\title{
Risk Factors, Clinical Pattern and Outcome of Stroke in a Referral Hospital, Northwest Ethiopia
}

\author{
Ermias Shenkutie Greffie, Tadesse Mitiku, Seid Getahun
}

Department of Internal Medicine, University of Gondar, Gondar, Ethiopia

Email address:

ermiasshe@yahoo.com (E. S. Greffie), tade2003.mit@gmail.com (T. Mitiku), seidgech014@gmail.com (S. Getahun)

\section{To cite this article:}

Ermias Shenkutie Greffie, Tadesse Mitiku, Seid Getahun. Risk Factors, Clinical Pattern and Outcome of Stroke in a Referral Hospital, Northwest Ethiopia. Clinical Medicine Research. Vol. 4, No. 6, 2015, pp. 182-188. doi: 10.11648/j.cmr.20150406.13

\begin{abstract}
Stroke is one of the commonest causes of morbidity and mortality among non-communicable diseases. Its occurrence has been significantly increasing in Sub Saharan Africa in the last couple of decades. Mortality has been shown to be higher in this area compared to developed countries. Yet, there is a big information gap about stroke in Ethiopia. This study aimed at describing the clinical pattern, risk factors and outcome of adult stroke patients admitted to university of Gondar hospital. Retrospective chart record analysis of adult patients with stroke who had Computerized tomography(CT) scan of the brain admitted to University of Gondar hospital medical wards from June 2010 to May 2013 was done. Relevant data including sociodemographics, risk factors, type of stroke and outcome were collected using a data extraction form. Data was entered and analyzed using Epi info7. Results: A total of 98 patients with a median age of 68 years (IQR: 60-76) and F:M ratio of 1.13:1 were analyzed. Ischemic stroke accounted for $69.4 \%$ of the cases. The median ages of patients with hemorrhagic and ischemic stroke were 60 and 70 years respectively $(\mathrm{P}=0.0027)$. Hypertension $(55.9 \%)$, any type of structural heart disease $(44.6 \%)$ and atrial fibrillation $(28.7 \%)$ were the most commonly identified risk factors. Atrial fibrillation was more prevalent in ischemic stroke patients $(34.3 \%)$ compared to hemorrhagic stroke $(14.8 \%)(\mathrm{P}=0.049)$. In hospital mortality rate was $13 \%$ with median duration of hospital stay before death of 6 days. Respiratory failure secondary to aspiration pneumonia and increased intracranial pressure were the most common immediate causes of death. Conclusion: Even though ischemic stroke was the most common stroke subtype, the proportion of hemorrhagic stroke was higher compared to studies from the developed world. Appropriate screening and treatment of hypertension, structural heart disease and atrial fibrillation should be give due attention as they are the most commonly identified risk factors. Most deaths occurred early after admission due to stroke related acute complications with respiratory failure; as such an emergency stroke care unit capable of managing these complications can partially decrease the death rate.
\end{abstract}

Keywords: Cerebrovascular Accident, University of Gondar Hospital, in Hospital Mortality

\section{Introduction}

Stroke or cerebrovascular accident is defined clinically as a focal or global disturbance of cerebral function of sudden onset lasting 24 hours or longer or leading to death with no apparent cause other than that of vascular origin[1]. It used to be a common problem in developed countries as compared to third world countries in the past. Nevertheless, recent community surveys undertaken in African countries showed stroke prevalence between 200 and 300 per $100,000[2-4]$. Although these figures are lower when compared to reports from developed countries (600-1000 per 100,000), they are significantly higher than previous reports of stroke prevalence in African countries (58-68 per
100,000) [5-7]. The most recent estimated stroke incidence rate in Africa is 316 per 100,000 with age standardized prevalence of 981 per 100,000 population [8]. The crude mortality rate of stroke in a rural community was 114 per 100,000 in a study from South Africa [9]. In general, SubSaharan Africa (SSA) can be considered as undergoing epidemiologic transition where the increasing burden of non-communicable disease is competing with the already prevailing burden of infectious disease. Among the global deaths from stroke of all age in 2005, $87 \%$ occurred in lowincome and middle-income countries. In fact, this fraction of global stroke deaths increases when people under 70 years were considered [10]. The age-standardized stroke mortality is also significantly higher in SSA countries as compared to developed countries [5]. In general, stroke is 
associated with significant mortality at a relatively younger age in developing countries.

Most of the conventional risk factors for stroke described elsewhere are also reported at varying degrees in SSA. Hypertension is by far the most common risk factor identified in many studies in SSA being present in more than $80 \%$ of the cases. Most patient were either not diagnosed or not on treatment for their hypertension [1115]. Cardiac disease especially rheumatic heart disease with mitral valve involvement and atrial fibrillation is also a common risk factor for embolic stroke in younger patients $[13,16-18]$. Diabetes is also reported as a significant risk factor alone or together with hypertension. In an Ethiopian and Kenyan studies it was identified in $26 \%$ and $33 \%$ respectively $[11,12]$.

Despite the evidences showing increasing trend of stroke in SSA, good-quality data on the epidemiology, prevention, and management of stroke in SSA are still deficient. The situation in our country is also no different. Although stroke is currently observed to be one of the commonest reasons of admission in many Ethiopian hospitals, its prevalence in the community is not known as there are no recent community based studies. So far there is only one prevalence study by Reda et. al. which showed a low prevalence of stroke(15 per $100,000)$ in a rural community based study in Ethiopia during 1986-1989[19]. The age adjusted stroke mortality from WHO's report in 2004 is high estimated to be 122 and 153.8 per 100,000 for males and females respectively in Ethiopia [5]. There are only few local hospital based studies on this issue in Ethiopia [13, 16, 20].

As patients usually present late and the standard of care is poor compared to hospitals in developed countries, the in hospital mortality is expected to be higher. Most of the information regarding stroke mortality and its determinants that we use in the management of stroke comes from studies in developed countries. So it is imperative that a lot has to be done to address the issues concerning the burden of stroke, risk factors and its mortality in Ethiopia. A recent study conducted in our hospital showed that hemorrhagic stroke was common but did not assess the risk factors, clinical presentation and outcome of stroke. Therefore, this study aimed at determining the clinical pattern, in hospital case fatality and outcome of CT scan proven stroke patients admitted to University of Gondar Hospital.

\section{Methods}

\subsection{Study Design and Setting}

This is a retrospective study conducted in University of Gondar Hospital, a tertiary hospital located in Gondar town, Northwest Ethiopia. The hospital serves as a referral hospital for a catchment area of 5 million people. Department of Internal medicine, currently running residency training in internal medicine, has ten internists and one neurologist. Even though CT scan is available in the hospital, not all patients with stroke get a CT scan due to frequent malfunctioning of the machine and cost issue. Other investigation modalities including electrocardiography (ECG), echocardiography, basic hematology and chemistry tests are readily available.

We retrieved case records of patients with diagnosis of stroke admitted to university of Gondar hospital during a three year period (June 2010- May 2013). Patients who fulfill the World Health Organization (WHO) case definition of stroke and who had a CT scan report were included in the study while patients under the age of 18 years and those with incomplete case records were excluded[1]. The investigator and trained medical residents collected all relevant data including sociodemographics, risk factor profile, clinical presentation, laboratory results, pathologic subtype and outcome from the case records using a standardized data extraction form.

\subsection{Data Processing and Analysis}

Data was checked for completeness and accuracy and was entered to Epi Info 7. Data analysis was performed using the same software. Descriptive statistics is presented in means and standard deviation or medians with inter quartile range for numerical variables and frequency percentages for categorical variables. Group comparison was done using Chisquare for categorical variables and student $t$ test and ANOVA for continuous variables with normally distributed variance. Kruskal Wallis/Mann Whitney test was used for comparison of group means of variables with skewed distribution. Statistical significance was defined by $\mathrm{P}$ value less than 0.05 . Tables and graphs were used to show results as appropriate.

\subsection{Ethical Consideration}

This study required secondary data from medical case records and patients were not contacted. The data from the case records were handled with strong confidentiality. Neither the case records nor the data extracted were used for any other purpose. The research proposal was approved by be the ethical board of school of medicine of University of Gondar. The study was started after ethical clearance was obtained from the board and after permission from the hospital management to review records.

\section{Results}

A total of 270 case fulfilling WHO's criteria for stroke were retrieved from the record room. Amongst those CT scan reports were found in 104 patients. The CT scan performance rate was $38.5 \%$. Six patients were excluded due to incomplete data. Therefore, a total of 98 cases fulfilling the inclusion criteria were finally selected for analysis.

\subsection{Sociodemographic Characteristics}

Females and males comprised 52(53.1\%) and 46 (46.9\%) of the study population with a ratio of $1.13: 1$. The median age of the population was 68 years (IQR: 60-76). Patients 
from urban residence accounted for 55.4\% while those from rural area accounted for $44.6 \%$. Stroke in the young, defined by age less than 45 years, accounted for 7(7.1\%) of the total cases.

\subsection{Stroke Subtypes}

The most common stroke subtype was ischemic stroke accounting for $68(69.4 \%)$ cases while hemorrhagic stroke accounted for the remaining $30(30.6 \%)$ cases. Included among the hemorrhagic strokes is one case of subarachinoid hemorrhage. (Figure 1)

As shown in Figure 2 the median age of patients with ischemic stroke was 70 years (IQR: 60.5-78) compared to 60 years (IQR: 55-70)for hemorrhagic stroke $(\mathrm{P}=0.0027)$. There was no statistically significant difference among the two stroke subtypes with sex and area of residence.

\subsection{Risk Factors}

Table 1 describes the frequency of identified risk factors in the different stroke subtypes. Hypertension was the most common risk factor identified in $55(53.2 \%)$ of the cases. It was identified in $53 \%$ and $60 \%$ of patients with ischemic and hemorrhagic stroke respectively $(\mathrm{P}=0.65)$. Diabetes was identified in $12(12.4 \%)$ of the cases. Nine patients $(9.3 \%)$ had both hypertension and diabetes.

Any type of structural heart disease was present in $46(46.9 \%)$ of patients. Figure 3 shows that among the structural heart diseases degenerative valve disease and hypertensive heart disease were the most common. Rheumatic heart disease was not identified in any of the patients. Atrial fibrillation was present in $27(28.72 \%)$ of the cases. It was more common in ischemic stroke patients $(34.8 \%)$ compared to hemorrhagic stroke patients $(14.3 \%)$ $(\mathrm{P}=0.049)$. Seven patients $(7.2 \%)$, all with ischemic stroke, had a previous history of stroke. Only one patient had history of smoking. Among 80 patients tested for HIV, none were positive.

Table 1. Sociodemographic and risk factor profile of stroke patients admitted to University of Gondar Hospital during June 2010 to May 2013, Gondar, Ethiopia.

\begin{tabular}{|c|c|c|c|c|c|}
\hline Variables & & Total N(\%) & Ischemic N(\%) & Hemorrhagic N(\%) & P value \\
\hline \multirow{2}{*}{ Sex } & $\mathrm{F}$ & $52(53.1)$ & $36(52.9)$ & $16(53.3)$ & \multirow{2}{*}{1.0} \\
\hline & M & $46(46.9)$ & $32(47.1)$ & $14(46.7)$ & \\
\hline \multirow{3}{*}{$\operatorname{Age}(y r s)$} & $18-39$ & $3(3.1)$ & $0(0.0)$ & $3(10.0)$ & \multirow{3}{*}{0.003} \\
\hline & $40-64$ & $39(39.8)$ & $23(33.8)$ & $16(53.3)$ & \\
\hline & $>65$ & $56(57.1)$ & $45(66.2)$ & $11(36.7)$ & \\
\hline \multirow{2}{*}{$\begin{array}{l}\text { Area of residence } \\
(\mathrm{n}=92)\end{array}$} & Urban & $51(55.4)$ & $37(57.8)$ & $14(50.0)$ & \multirow{2}{*}{0.5} \\
\hline & Rural & $41(44.6)$ & $27(42.2)$ & $14(50.0)$ & \\
\hline \multirow{2}{*}{$\begin{array}{l}\text { Hypertension } \\
(\mathrm{n}=96)\end{array}$} & yes & $53(55.2)$ & $35(55)$ & $18(60)$ & \multirow{2}{*}{0.66} \\
\hline & no & $43(44.8)$ & $31(47)$ & $12(40)$ & \\
\hline \multirow{2}{*}{$\begin{array}{l}\mathrm{DM} \\
(\mathrm{n}=95)\end{array}$} & yes & $12(12.6)$ & 11(16.7) & $1(3.4)$ & \multirow{2}{*}{0.1} \\
\hline & no & $83(87.4)$ & $55(83.30$ & $28(96.6)$ & \\
\hline \multirow{2}{*}{$\begin{array}{l}\text { Structural heart disease } \\
(\mathrm{n}=98)\end{array}$} & yes & $46(46.9)$ & $31(45.6)$ & $15(50.0)$ & \multirow{2}{*}{0.83} \\
\hline & no & $52(53.1)$ & $37(54.4)$ & $15(50.0)$ & \\
\hline \multirow{2}{*}{$\begin{array}{l}\text { Atrial fibrillation } \\
(\mathrm{n}=94)\end{array}$} & Yes & $27(28.7)$ & $23(34.8)$ & $4(14.3)$ & \multirow{2}{*}{0.049} \\
\hline & no & $67(71.3)$ & $43(65.2)$ & $24(85.7)$ & \\
\hline \multirow{2}{*}{$\begin{array}{l}\text { Previous stroke } \\
(\mathrm{n}=97)\end{array}$} & Yes & $7(7.2)$ & $7(10.4)$ & $0(0.0)$ & \multirow{2}{*}{0.1} \\
\hline & No & $90(92.8)$ & $60(89.6)$ & $30(100)$ & \\
\hline \multirow{2}{*}{$\begin{array}{l}\text { Total cholesterol(mg/dl) } \\
(\mathrm{n}=77)\end{array}$} & $>200$ & $4(5.2)$ & $2(3.6)$ & $2(9.5)$ & \multirow{2}{*}{0.3} \\
\hline & $<200$ & $73(94.8)$ & $54(96.4)$ & $19(90.5)$ & \\
\hline
\end{tabular}

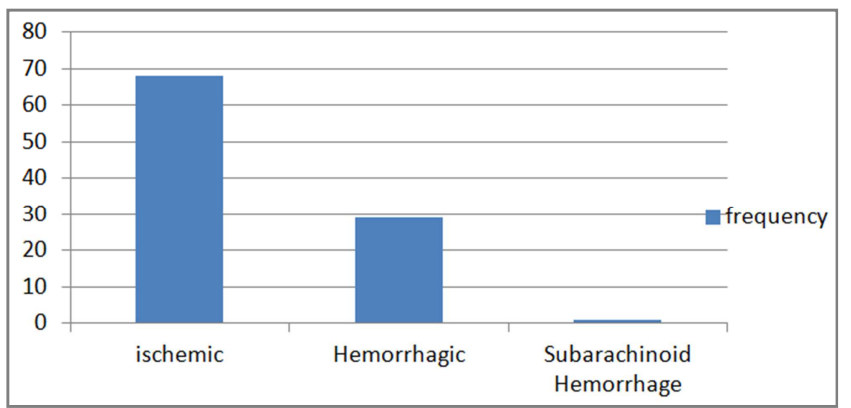

Figure 1. Frequency of stroke subtypes among patients admitted with stroke to University of Gondar Hospital during June 2010 to May 2013, Gondar, Ethiopia.

\subsection{Neurologic Deficits}

Table 2 shows the frequency distribution of different neurologic deficits identified in our study patients. Hemiparesis/hemipegia (92.39\%) and cranial nerve deficits $(60.20 \%)$ were the most common neurologic deficits. Overall, alteration of consciousness was present in $28(28.6 \%)$ of patients. Urinary incontinence was present in $9(9.1 \%)$ patients. Seizure occurred in $10(10.2 \%)$ patients.

\subsection{Laboratory Abnormalities}

Carotid Doppler ultrasound studies were done for a total of 20 patients. It was normal in 5(25\%), showed atherosclerosis without stenosis in $10(50 \%)$ and atherosclerosis with stenosis in $5(25 \%)$ patients. Anemia 
(hemoglobin $<12 \mathrm{~g} / \mathrm{dl})$ and thrombocytopenia $(<150000$ cells $/ \mathrm{mm}^{3}$ ) were found in $14.94 \%$ and $14.77 \%$ of patients with available results. Hyperglycemia (random blood sugar $>180 \mathrm{mg} / \mathrm{dl}$ ) and deranged renal function (creatinine $>1.2 \mathrm{mg} / \mathrm{dl}$ ) occurred in $12.5 \%$ and $15.7 \%$.

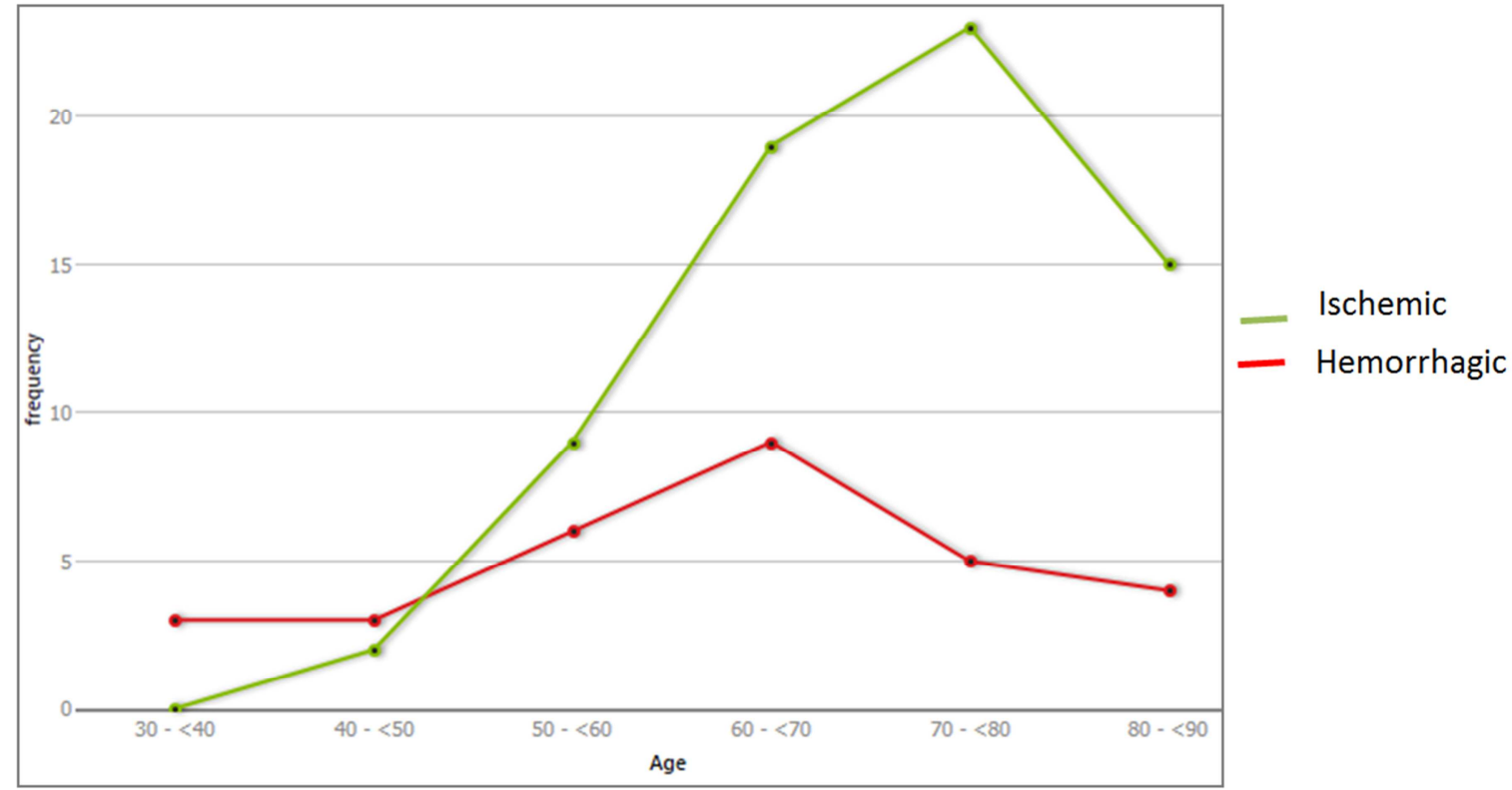

Figure 2. Age distribution of patients with ischemic and hemorrhagic stroke admitted to University of Gondar Hospital during June 2010 to May 2013, Gondar Ethiopia.

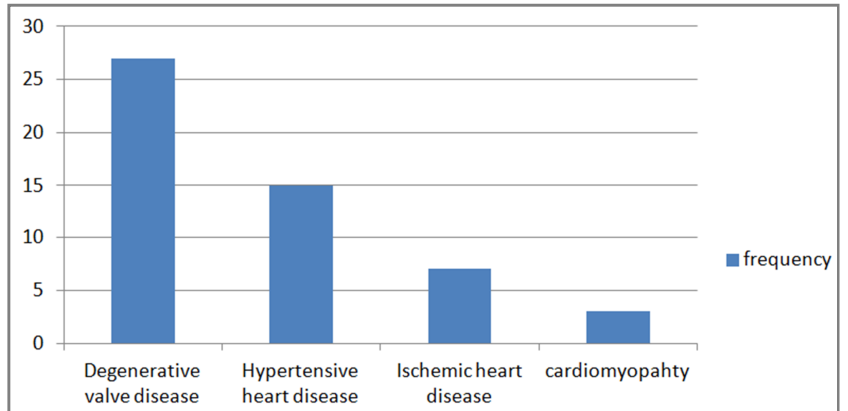

Figure 3. Types of structural heart disease in patients with stroke admitted to University of Gondar Hospital during June 2010 to May 2013, Gondar, Ethiopia.

Table 2. Neurologic deficits in patients with stroke admitted to University of Gondar Hospital during June 2010 to May 2013, Gondar, Ethiopia.

\begin{tabular}{|c|c|c|c|c|}
\hline & $\begin{array}{l}\text { Total } \\
\text { N(\%) } \\
\end{array}$ & $\begin{array}{l}\text { Ischemic } \\
\mathbf{N}(\%)\end{array}$ & $\begin{array}{l}\text { Hemorrhagic } \\
\mathrm{N}(\%)\end{array}$ & $\begin{array}{l}\mathbf{P} \\
\text { value }\end{array}$ \\
\hline Hemiparesis/plegia & 92(93.9) & $65(95.6)$ & $27(90.0)$ & 0.37 \\
\hline Cranial nerve palsy & $59(60.2)$ & $40(58.8)$ & $19(63.3)$ & 0.83 \\
\hline $\begin{array}{l}\text { Alteration of } \\
\text { consciousness }\end{array}$ & $28(28.6)$ & $16(23.5)$ & $12(40)$ & 0.14 \\
\hline $\begin{array}{l}\text { Urinary } \\
\text { incontinence }\end{array}$ & $9(9.2)$ & $4(5.9)$ & $5(16.7)$ & 0.13 \\
\hline seizure & $10(10.2)$ & $7(10.3)$ & $3(10.0)$ & 1.0 \\
\hline
\end{tabular}

\subsection{Outcome}

The in hospital case fatality of stroke was $13.3 \%$. Fgure 4 shows that among the 98 patients with documented outcomes, 58(59.18\%) were discharged with improvement whereas $13(13.3 \%)$ died in the hospital. Eight (8.2\%) patients were discharged with the same condition and one was referred. Eighteen (18.3\%) patients were discharged against medical advice on self and family request. (Figure 4) The median duration of hospital stay was 13(IQR: 7 -19) days and ranged from 1 to 60 days. The median duration of hospital stay before death was 6 days. The commonest complications identified were aspiration pneumonia and increased intracranial pressure. Table 3 shows the associated complications and concomitant diagnoses. The most commonly attributed cause of death was respiratory failure secondary to aspiration pneumonia in 5 patients and increased intracranial pressure in 3 patients. (Table 4)

Table 3. Complications in patients with stroke admitted to University of Gondar Hospital during June 2010 to May 2013, Gondar, Ethiopia.

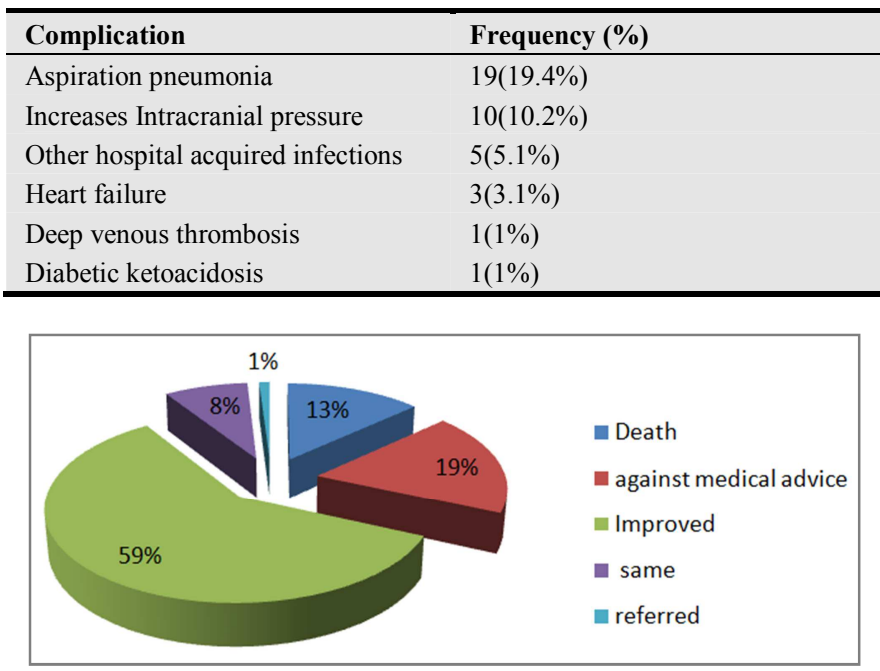

Figure 4. Outcome of patients with stroke admitted to University of Gondar Hospital during June 2010 to May 2013, Gondar, Ethiopia. 
Table 4. Attributable cause of death in patients with stroke admitted to University of Gondar Hospital during June 2010 to May 2013, Gondar, Ethiopia.

\begin{tabular}{ll}
\hline Cause of Death & Frequency (\%) \\
\hline Aspiration pneumonia & $5(38.5)$ \\
Increased ICP & $3(23.0)$ \\
Unknown & $5(38.5)$ \\
\hline
\end{tabular}

\section{Discussion}

This is the first study on stroke in University of Gondar Hospital focusing on the risk factors, clinical pattern and outcome. The CT scan performance rate $(38.5 \%)$ was very low and as this study included only patients with CT scan reports it may not reflect the true nature of stroke in our patients.

In our study ischemic stroke was the commonest subtype accounting for $69.4 \%$ of cases. There is a great variation in this proportion in different SSA studies which utilized CT scan with reported rates for hemorrhagic stroke ranging from $15-60 \%[11,12,15,17,21]$. Despite this difference, hemorrhagic stroke appears to be more common in SSA countries compared to the developed world. This difference may be the result of difference in study designs, hospital admission bias or difference in the population pyramid, socioeconomic and risk factor profiles between the two populations.

The age distribution observed in our study(68 years) was higher than results from similar hospital based studies in other SSA countries showing median ages less than 60 years $[11,13,14,22]$. It was rather consistent with reports from developed countries. This disagreement is an indication that hospital based studies are liable to admission bias and community based studies are required to clearly find out the age distribution of stroke in our area. This study has shown that patients with hemorrhagic stroke were younger (60 years) than those with ischemic stroke ( 70 years). This seems to be the effect of higher prevalence of hypertension in this group and reflects the earlier onset of end organ damage due to undertreated hypertension.

Our study, similar to other studies elsewhere, has shown that hypertension, found in more than half, is by far the most commonly identified risk factor. Even though we haven't assessed the treatment history, our experience and other studies from SSA tells us that most patients with hypertension are either previously undiagnosed or are not appropriately treated [11-15]. Diabetes was identified in $10 \%$ of our patients which is lower than studies elsewhere [11, 12]. It was often present together with hypertension which entails their synergistic effect on stroke. Therefore, the beneficial effect of screening and treatment of hypertension and diabetes for the prevention of stroke cannot be overemphasized.

Atrial fibrillation was identified in $28.7 \%$ of all cases and $34.8 \%$ of ischemic stroke cases in our study which is higher than reports from elsewhere (15-23\%) [23-25]. As the diagnosis was based on a single ECG, the prevalence could be underestimated. Despite the fact that anticoagulants are effective for preventing stroke from atrial fibrillation, none of the patients were taking anticoagulants. Rheumatic heart disease, which was reported to be a common risk factor for stroke especially in the young in other Ethiopian studies, was found to be less common in our study.

Tobacco smoking, as shown in other Ethiopian studies was very rare in our patients while it was common in other countries (13.4\%-26.8\%) [15, 26-29]. Although, fasting lipid profiles were not available in our cases, none of the patients had previous diagnosis of dyslipidemia and high cholesterol was measured only in $5.2 \%$.

HIV was associated with stroke in some studies but its prevalence in our patients was low [30, 31]. Low prevalence of HIV in stroke patients was also reported from other studies in Ethiopia and Kenya [12, 26].

The in hospital case fatality rate of stroke in our study, $13 \%$, while higher than reports from western studies, is quite lower compared to similar SSA studies reporting rates between $14.7 \%-44.5 \%[11,13,15,27,28]$. This difference at least partly is explained by the high rate of discharge against medical advice $(30 \%)$ in our patients. As it is our experience that patient attendants make this decision when patient's condition is deteriorating or is not changing, we believe that the mortality rate is higher than what is reported here. Thus, the in hospital mortality rate may not reflect the true short term mortality. A prospective follow up study with confirmation is necessary to get the true short term mortality. Most of the deaths were attributed to early complications of stroke especially increased intracranial pressure and aspiration pneumonia. Prevention, early identification and management of these factors would at least have salvaged some of the patients. Therefore, the increased early mortality in our patients is partly due to the absence of an emergency stroke care setup capable of early identification and management of these acute complications.

\section{Conclusion}

There was a low rate of CT scan performance rate. Ischemic stroke was the most common type of stroke. Nonetheless, hemorrhagic stroke occurred more frequently compared to hospitals in developed countries. Patients with hemorrhagic stroke were younger than those with ischemic stroke. Hypertension and atrial fibrillation were the most prevalent risk factors. Tobacco smoking and dyslipidemia were not common risk factors. The in hospital case fatality rate $(13 \%)$ is lower than other SSA hospital studies but significant proportion of patients were discharged against medical advice (19\%). The most common attributable causes of death were aspiration pneumonia and increased intracranial pressure.

\section{Recommendation}

A prospective hospital based study and community based stroke incidence and prevalence studies are required to define 
the true sociodemographic characteristics of stroke and the associated factors in our population. Strategies for screening and management of hypertension, structural heart disease and atrial fibrillation should be given priority as these are the most prevalent risk factors identified. An emergency care set up, perhaps protocol based, capable of early brain imaging, identifying and managing early stroke complications is invaluable for the prevention of early stroke related mortality.

\section{Limitations of the Study}

This is a hospital based study and as such the results cannot be generalized to the general population. As this study is based on retrospective chart review, the data obtained was not complete. The low rate of CT scan performance has limited our sample size. Significant number of patients were discharged against medical advice which made the in hospital mortality non representative of the short term mortality. The study hasn't assessed the rate of disability and functional limitation.

\section{Abbreviations}

\author{
CT: Computerized tomography \\ DM: Diabetes mellitus \\ ECG: Electrocardiography \\ HIV: Human immunodeficiency virus \\ SSA: Sub Saharan Africa; \\ WHO: World Health Organization
}

\section{References}

[1] Feigin, V.L., et al., Stroke epidemiology: a review of population-based studies of incidence, prevalence, and casefatality in the late 20th century. Lancet neurology, 2003. 2(1): p. 43-53.

[2] Connor, M.D., et al., Prevalence of stroke survivors in rural South Africa: results from the Southern Africa Stroke Prevention Initiative (SASPI) Agincourt field site. Stroke, 2004. 35(3): p. 627-32.

[3] Balogou, A.A., A. Doh, and K.E. Grunitzky, [Neurological disorders and endemic goiter: comparative analysis of 2 provinces in Togo]. Bull Soc Pathol Exot, 2001. 94(5): p. 40610 .

[4] Walker, R., et al., Stroke incidence in rural and urban Tanzania: a prospective, community-based study. The Lancet Neurology, 2010. 9(8): p. 786-792.

[5] WHO. WHO Global InfoBase. 2004 [cited 2013 July 20]; Available from: http://www.who.int/infobase

[6] Matenga, J., Stroke incidence rates among black residents of Harare-a prospective community-based study. SOUTH AFRICAN MEDICAL JOURNAL-CAPE TOWN-MEDICAL ASSOCIATION OF SOUTH AFRICA-, 1997. 87: p. 606-608.

[7] Osuntokun, B., et al., Neurological disorders in Nigerian Africans: a community - based study. Acta neurologica scandinavica, 1987. 75(1): p. 13-21.
[8] Owolabi, M.O., et al., The burden of stroke in Africa: a glance at the present and a glimpse into the future: review article. 2015.

[9] Maredza, M., M.Y. Bertram, and S.M. Tollman, Disease burden of stroke in rural South Africa: an estimate of incidence, mortality and disability adjusted life years. BMC neurology, 2015. 15(1): p. 54.

[10] Strong, K., C. Mathers, and R. Bonita, Preventing stroke: saving lives around the world. Lancet Neurol, 2007. 6(2): p. 182-7.

[11] Asefa, G. and S. Meseret, CT and clinical correlation of stroke diagnosis, pattern and clinical outcome among stroke patients visting Tikur Anbessa Hospital. Ethiop Med J, 2010. 48(2): p. 117-22.

[12] Jowi, J. and P. Mativo, Pathological Sub-Types, Risk Factors And Outcome Of Stroke At The Nairobi Hospital, Kenya. East African Medical Journal, 2008. 85(12).

[13] Zenebe, G., M. Alemayehu, and J. Asmera, Characteristics and outcomes of stroke at Tikur Anbessa Teaching Hospital, Ethiopia. Ethiop Med J, 2005. 43(4): p. 251-9.

[14] Kengne, A.P. and C.S. Anderson, The neglected burden of stroke in Sub-Saharan Africa. Int J Stroke, 2006. 1(4): p. 18090 .

[15] Deresse, B. and D. Shaweno, Epidemiology and in-hospital outcome of stroke in South Ethiopia. Journal of the neurological sciences, 2015. 355(1): p. 138-142.

[16] Alemayehu, B. and K. Oli, Stroke Admission to Tikur Anbassa Teaching Hospital: With Emphasis on Stroke in the Young. Ethiopian Journal of Health Development, 2002. 16(3): p. 309-315.

[17] Joubert, J., The MEDUNSA Stroke Data Bank. An analysis of 304 patients seen between 1986 and 1987. S Afr Med J, 1991. 80(11-12): p. 567-70.

[18] Matenga, J., I. Kitai, and L. Levy, Strokes among black people in Harare, Zimbabwe: results of computed tomography and associated risk factors. Br Med J, 1986. 292(6536): p. 164951.

[19] Tekle-Haimanot, R., et al., Community-based study of neurological disorders in rural central Ethiopia. Neuroepidemiology, 1990. 9(5): p. 263-77.

[20] Kebede, B., Stroke Admissions to Tikur Anbessa Hospital, With Emphasis on Stroke in The Young Ethio.J. Health. Dev., 2002. 16(3): p. 309-315.

[21] Nyame, P.K., K.B. Jumah, and S. Adjei, Computerised tomographic scan of the head in evaluation of stroke in Ghanaians. East Afr Med J, 1998. 75(11): p. 637-9.

[22] Walker, R.W., et al., Mortality and recovery after stroke in the Gambia. Stroke, 2003. 34(7): p. 1604-9.

[23] Lamassa, M., et al., Characteristics, Outcome, and Care of Stroke Associated With Atrial Fibrillation in Europe Data From a Multicenter Multinational Hospital-Based Registry (The European Community Stroke Project). Stroke, 2001. 32(2): p. 392-398.

[24] Jorgensen, H.S., et al., Acute stroke with atrial fibrillation. The Copenhagen Stroke Study. Stroke, 1996. 27(10): p. 1765-9. 
[25] Sandercock, P., et al., Atrial fibrillation and stroke: prevalence in different types of stroke and influence on early and long term prognosis (Oxfordshire community stroke project). BMJ, 1992. 305(6867): p. 1460-5.

[26] Alemayehu, C.M. and S.K. Birhanesilasie, Assessment of stoke patients: Occurrence of unusually high number of haemorrhagic stroke cases in Tikur Anbessa Specialized Hospital, Addis Ababa, Ethiopia.

[27] Atadzhanov, M., et al., Stroke Characteristics and Outcomes of Adult Patients Admitted to the University Teaching Hospital, Lusaka, Zambia. Open General and Internal Medicine Journal, 2012. 5: p. 3-8.

[28] Desalu, O.O., et al., A review of stroke admissions at a tertiary hospital in rural Southwestern Nigeria. Ann Afr Med, 2011. 10(2): p. $80-5$.

[29] Sridharan, S.E., et al., Incidence, types, risk factors, and outcome of stroke in a developing country the trivandrum stroke registry. Stroke, 2009. 40(4): p. 1212-1218.

[30] Mlay, M. and M. Bakari, The prevalence of HIV among patients admitted with stroke at the Muhimbili National Hospital, Dar es Salaam, Tanzania. Tanzania Journal of Health Research, 2010. 12(2): p. 105-113.

[31] Chow, F.C., et al., Comparison of Ischemic Stroke Incidence in HIV-Infected and Non-HIV-Infected Patients in a US Health Care System. JAIDS Journal of Acquired Immune Deficiency Syndromes, 2012. 60(4): p. 351-358. 